\title{
POLYNOMIAL FIRST INTEGRALS FOR WEIGHT-HOMOGENEOUS PLANAR POLYNOMIAL DIFFERENTIAL SYSTEMS OF WEIGHT DEGREE 4
}

\author{
JAUME LLIBRE AND CLAUDIA VALLS
}

\begin{abstract}
We classify all of the weight-homogeneous planar polynomial differential systems of weight degree 4 having a polynomial first integral.
\end{abstract}

1. Introduction and statement of the main result. In this paper, we deal with polynomial differential systems of the form:

$$
\frac{d \mathbf{x}}{d t}=\dot{\mathbf{x}}=\mathbf{P}(\mathbf{x}), \quad \mathbf{x}=(x, y) \in \mathbb{C}^{2},
$$

with $\mathbf{P}(\mathbf{x})=\left(P_{1}(\mathbf{x}), P_{2}(\mathbf{x})\right)$ and $P_{i} \in \mathbb{C}[x, y]$ for $i=1,2$. As usual, $\mathbb{Q}^{+}$, $\mathbb{R}$ and $\mathbb{C}$ will denote the sets of non-negative rational, real and complex numbers, respectively, and $\mathbb{C}[x, y]$ denotes the polynomial ring over $\mathbb{C}$ in the variables $x, y$. Here, $t$ is real or complex.

System (1.1) is weight homogeneous or quasi-homogeneous if there exist $\mathbf{s}=\left(s_{1}, s_{2}\right) \in \mathbb{N}^{2}$ and $d \in \mathbb{N}$ such that, for arbitrary $\alpha \in \mathbb{R}^{+}=$ $\{a \in \mathbb{R}, a>0\}$,

$$
P_{i}\left(\alpha^{s_{1}} x, \alpha^{s_{2}} y\right)=\alpha^{s_{i}-1+d} P_{i}(x, y),
$$

for $i=1,2$. We call $\mathbf{s}=\left(s_{1}, s_{2}\right)$ the weight exponent of system (1.1) and $d$ the weight degree with respect to the weight exponent $\mathbf{s}$. In the particular case where $\mathbf{s}=(1,1)$, system (1.1) is called a homogeneous polynomial differential system of degree $d$.

2010 AMS Mathematics subject classification. Primary 34A05, 34A34, 34C14.

Keywords and phrases. Polynomial first integrals, weight-homogeneous polynomial differential systems.

The first author is partially supported by MINECO/FEDER, grant Nos. MTM2008-03437 and MTM2013-40998-P, ICREA Academia, grant Nos. FP7PEOPLE-2012-IRSES 318999 and 316338, AGAUR, grant No. 2014SGR-568, and grant No. UNAB13-4E-1604. The second author is supported by Portuguese national funds through FCT, Fundação para a Ciência e a Tecnologia, grant No. PEstOE/EEI/LA0009/2013 (CAMGSD).

Received by the editors on October 9, 2012, and in revised form on January 9, 2015 . 
Recently, such systems have been investigated by several authors. Labrunie [12] and Ollagnier [15] characterized all polynomial first integrals of the three-dimensional $(a, b, c)$ Lotka-Volterra systems. Maciejewski and Strelcyn [14] proved that the so-called Halphen system has no algebraic first integrals. However, some of the best results for general weight homogeneous polynomial differential systems have been provided by Furta [10] and Goriely [11]. For quadratic homogeneous polynomial differential systems, we refer the reader to $[\mathbf{1 3}, \mathbf{1 6}]$. Additionally, we refer the reader to $[\mathbf{1}-\mathbf{5}]$.

A non-constant function $H(x, y)$ is a first integral of system (1.1) if it is constant on all solution curves $(x(t), y(t))$ of system (1.1), i.e., $H(x(t), y(t))$ is constant for all values of $t$ for which the solution $(x(t), y(t))$ is defined. If $H$ is $C^{1}$, then $H$ is a first integral of system (1.1) if and only if

$$
P_{1} \frac{\partial H}{\partial x}+P_{2} \frac{\partial H}{\partial y}=0 .
$$

The function $H(x, y)$ is weight homogeneous of weight degree $m$ with respect to the weight exponent $\mathbf{s}$ if it satisfies $H\left(\alpha^{s_{1}} x, \alpha^{s_{2}} y\right)=$ $\alpha^{m} H(x, y)$, for all $\alpha \in \mathbb{R}^{+}$.

Given $H \in \mathbb{C}[x, y]$, we can split it into the form

$$
H=H_{m}+H_{m+1}+\cdots+H_{m+l},
$$

where $H_{m+i}$ is a weight homogeneous polynomial of weight degree $m+i$ with respect to the weight exponent s, i.e.,

$$
H_{m+i}\left(\alpha^{s_{1}} x, \alpha^{s_{2}} y\right)=\alpha^{m+i} H_{m+i}(x, y) .
$$

The following well-known proposition (see [13] for proof) reduces the study of the existence of analytic first integrals of a weighthomogeneous polynomial differential system (1.1) to the study of the existence of a weight-homogeneous polynomial first integral.

Proposition 1.1. Let $H$ be an analytic function, and let

$$
H=\sum_{i} H_{i}
$$

be its decomposition into weight-homogeneous polynomials of weight degree $i$ with respect to the weight exponent $\mathbf{s}$. Then, $H$ is an analytic first 
integral of the weight-homogeneous polynomial differential system (1.1) if and only if each weight-homogeneous part $H_{i}$ is a first integral of system (1.1) for all $i$.

The main goal of this paper is to classify all analytic first integrals of the weight-homogenous planar polynomial differential systems of weight degree 4. In view of Proposition 1.1, we only need to classify all polynomial first integrals of the weight-homogenous planar polynomial differential systems of weight degree 4 . The classification of all polynomial first integrals (and hence, of all analytic first integrals) of the weight-homogenous planar polynomial differential systems of weight degree 1 is straightforward and trivial. The classification of all polynomial first integrals (and hence, of all analytic first integrals) of the weighthomogenous planar polynomial differential systems of weight degree 2 was given in $[\mathbf{8}, \mathbf{1 3}]$ and for systems of weight degree 3 in $[\mathbf{6}, \mathbf{8}]$.

Kowalevskaya exponents are used in the classification of all polynomial first integrals for weight-homogenous planar polynomial differential systems of weight degrees 2 and 3 . However, it was shown in [6, Theorem 4] that these exponents are useless for classifying the polynomial first integrals for weight-homogenous planar polynomial differential systems of weight degrees larger than 3 .

Proposition 1.2. The systems with weight degree 4 in $\mathbb{C}^{2}$ and their corresponding values of $\mathbf{s}$ can be written as follows:

$$
\begin{aligned}
\mathbf{s}=(1,1): & \dot{x}=a_{40} x^{4}+a_{31} x^{3} y+a_{22} x^{2} y^{2}+a_{13} x y^{3}+a_{04} y^{4}, \\
& \dot{y}=b_{40} x^{4}+b_{31} x^{3} y+b_{22} x^{2} y^{2}+b_{13} x y^{3}+b_{04} y^{4} ; \\
\mathbf{s}=(1,2): \dot{x}=a_{40} x^{4}+a_{21} x^{2} y+a_{02} y^{2}, & \\
\dot{y}=b_{50} x^{5}+b_{31} x^{3} y+b_{12} x y^{2} ; & \\
\mathbf{s}=(1,3): \dot{x}=a_{40} x^{4}+a_{11} x y, & \dot{y}=b_{60} x^{6}+b_{31} x^{3} y+b_{02} y^{2} ; \\
\mathbf{s}=(1,4): \dot{x}=a_{40} x^{4}+a_{01} y, & \dot{y}=b_{70} x^{7}+b_{31} x^{3} y ; \\
\mathbf{s}=(2,3): \dot{x}=a_{11} x y, & \dot{y}=b_{30} x^{3}+b_{02} y^{2} ; \\
\mathbf{s}=(2,5): \dot{x}=a_{01} y, & \dot{y}=b_{40} x^{4} \\
\mathbf{s}=(3,3): \dot{x}=a_{20} x^{2}+a_{11} x y+a_{02} y^{2}, & \dot{y}=b_{20} x^{2}+b_{11} x y+b_{02} y^{2} ; \\
\mathbf{s}=(6,9): \dot{x}=a_{01} y, & \dot{y}=b_{20} x^{2} .
\end{aligned}
$$


The proof of Proposition 1.2 is provided in Section 2.

In what follows, we state our main result, i.e., we classify when the systems of Proposition 1.2 exhibit a polynomial first integral. The systems with weight exponent $(1,1)$ having a polynomial first integral are given in Section 3. The systems with weight exponent $(3,3)$ having a polynomial first integral are studied inside the systems with weight exponent $(1,2)$. The polynomial first integrals for the other systems of Proposition 1.2 are provided in this introduction.

We introduce the change $(X, Y)=\left(x^{2}, y\right)$ in the planar weight homogeneous polynomial differential systems (1.1) of weight degree 4 with weight exponent $(1,2)$. With these new variables $(X, Y)$ the system with weight exponent $(1,2)$ becomes, after introducing the new independent variable $d \tau=x d t$,

$$
\begin{aligned}
& X^{\prime}=2 a_{40} X^{2}+2 a_{21} X Y+2 a_{02} Y^{2}, \\
& Y^{\prime}=b_{50} X^{2}+b_{31} X Y+b_{12} Y^{2},
\end{aligned}
$$

where the prime denotes the derivative with respect to $\tau$.

System (1.4) is a homogeneous quadratic planar polynomial system with $\mathbf{s}=(1,1)$. It is well known (see [9]) that, for each quadratic homogeneous system, there exist some linear transformation and a rescaling of time which transform system (1.4) into systems in (1.5).

$$
\begin{array}{lll}
\dot{x}=-2 x y+\frac{2}{3} x\left(p_{1} x+p_{2} y\right), & \dot{y}=-x^{2}+y^{2}+\frac{2}{3} y\left(p_{1} x+p_{2} y\right), \\
\dot{x}=-2 x y+\frac{2}{3} x\left(p_{1} x+p_{2} y\right), & \dot{y}=x^{2}+y^{2}+\frac{2}{3} y\left(p_{1} x+p_{2} y\right), \\
\dot{x}=-x^{2}+\frac{2}{3} x\left(p_{1} x+p_{2} y\right), & \dot{y}=2 x y+\frac{2}{3} y\left(p_{1} x+p_{2} y\right), \\
\dot{x}=\frac{2}{3} x\left(p_{1} x+p_{2} y\right), & \dot{y}=x^{2}+\frac{2}{3} y\left(p_{1} x+p_{2} y\right), \\
\dot{x}=\frac{2}{3} x\left(p_{1} x+p_{2} y\right), & \dot{y}=\frac{2}{3} y\left(p_{1} x+p_{2} y\right) .
\end{array}
$$

We prove the following theorem which characterizes all polynomial first integrals for the systems in (1.5).

Theorem 1.3. The homogeneous polynomial systems in (1.5) have a polynomial first integral $H$ if and only if one of the following conditions hold.

(a) The first system in (1.5) with $p_{1}=0, p_{2}=3(1-q) /(1+2 q)$ with $q=n / m \in \mathbb{Q}^{+}$and, in this case, $H=x^{m}\left(3 y^{2}-x^{2}\right)^{n}$. 
(b) The second system in (1.5) with $p_{1}=0, p_{2}=3(1-q) /(1+2 q)$ with $q=n / m \in \mathbb{Q}^{+}$and, in this case, $H=x^{m}\left(3 y^{2}+x^{2}\right)^{n}$.

(c) The third system, in (1.5) with $p_{1}=0$ and $p_{2}=3(1-2 q) /(2(1+q))$ with $q=n / m \in \mathbb{Q}^{+}$and, in this case, $H=x^{m} y^{n}$.

(d) The fourth system in (1.5) with $p_{1}=p_{2}=0$ and, in this case, $H=x$.

We note that systems with weight exponent $(3,3)$ coincide with systems (1.4), and hence, it can be written into systems in (1.5). Therefore, Theorem 1.3 applies to those systems.

The proof of Theorem 1.3 is given in Section 4 .

Theorem 1.4. The weight homogeneous polynomial differential systems with weight exponent $(1,3)$ and weight degree 4 have a polynomial first integral $H$ if and only if the following conditions hold:

(a) $a_{11}=a_{40}=0$ with $H=x$.

(b) $b_{60}=b_{31}=b_{02}=0$ with $H=y$.

(c) $\left(3 a_{11}-b_{02}\right)\left(3 a_{11}-2 b_{02}\right) \neq 0, a_{40}=-a_{11} b_{31} /\left(3 a_{11}-2 b_{02}\right)$, $3 a_{11} /\left(6 a_{11}-2 b_{02}\right)=m / n \in \mathbb{Q}^{+}$and $m / n<1$ with

$$
\begin{aligned}
H= & x^{3(n-m)}\left(\left(3 a_{11}-2 b_{02}\right) b_{60} x^{6}+2\left(3 a_{11}-b_{02}\right) b_{31} x^{3} y\right. \\
& \left.-\left(9 a_{11}^{2}-9 a_{11} b_{02}+2 b_{02}^{2}\right) y^{2}\right)^{m} .
\end{aligned}
$$

(d) $b_{31} / a_{40}=-m / n$ and $m / n \in \mathbb{Q}^{+}$with $H=x^{3 m}\left(b_{60} x^{3}+\left(b_{31}-\right.\right.$ $\left.\left.3 a_{40}\right) y\right)^{3 n}$.

(e) $b_{02}=0, a_{11} \neq 0, a_{40}=-b_{31} / 3$ and $b_{06}=-\left(3 a_{40}-b_{31}\right)^{2} /\left(12 a_{11}\right)$ with $H=b_{31} x^{3}-3 a_{11} y$.

(f) $\left(3 a_{11}-b_{02}\right)\left(3 a_{11}-2 b_{02}\right) \neq 0, a_{40}=-a_{11} b_{31} /\left(3 a_{11}-2 b_{02}\right), b_{06}=$ $-\left(3 a_{40}-b_{31}\right)^{2} /\left(4\left(3 a_{11}-b_{02}\right)\right), b_{02} \neq 0$ and $-3 a_{11} / b_{02}=n / m \in \mathbb{Q}^{+}$, with

$$
H=x^{3 n}\left(b_{31} x^{3}+\left(2 b_{02}-3 a_{11}\right) y\right)^{m} .
$$

The proof of Theorem 1.4 is given in Section 5 .

Theorem 1.5. The weight homogeneous polynomial differential systems with weight exponent $(1,4)$ and weight degree 4 have a polynomial first integral $H$ if and only if the following conditions hold: 
(a) $a_{40}=a_{01}=0$ and $H=x$.

(b) $b_{70}=b_{31}=0$ and $H=y$.

(c) $b_{31}=-4 a_{40}$, and $4 a_{40}^{2}+a_{01} b_{70} \neq 0$ with $H=-b_{70} x^{8}+8 a_{40} x^{4} y+$ $4 a_{01} y^{2}$.

(d) $b_{31}=-4 a_{40}, a_{40} b_{70} \neq 0$ and $4 a_{40}^{2}+a_{01} b_{70}=0$ with $H=$ $b_{70} x^{4}-4 a_{40} y$.

The proof of Theorem 1.5 is given in Section 6 .

Theorem 1.6. The weight homogeneous polynomial differential systems with weight exponent $(2,3)$ and weight degree 4 have a polynomial first integral $H$ if and only if $a_{11}=0$, in which case $H=x$, or $b_{30}=b_{02}=0$, in which case, $H=y$, or $a_{11}\left(3 a_{11}-2 b_{02}\right) \neq 0$ and $-2 b_{02} / a_{11}=n / m \in \mathbb{Q}^{+}$, in which case,

$$
H=x^{n}\left(2 b_{30} x^{3}-3 a_{11} y^{2}+2 b_{02} y^{2}\right)^{m} .
$$

The proof of Theorem 1.6 is given in Section 7 .

Theorem 1.7. The weight homogeneous polynomial differential systems with weight exponent $(2,5)$ and weight degree 4 have the polynomial first integral $H=2 b_{40} x^{5}-5 a_{01} y^{2}$.

The proof of Theorem 1.7 is given in Section 8 .

Theorem 1.8. The weight homogeneous polynomial differential systems with weight exponent $(6,9)$ and weight degree 4 have the polynomial first integral $H=2 b_{20} x^{3}-3 a_{01} y^{2}$.

The proof of Theorem 1.8 is given in Section 9 .

2. Proof of Proposition 1.2. From the definition of weight homogeneous polynomial differential systems (1.1) with weight degree 4 , the exponents $u_{i}$ and $v_{i}$ of any monomial $x^{u_{i}} y^{v_{i}}$ of $P_{i}$ for $i=1,2$, are such that they satisfy respectively the relations

$$
s_{1} u_{1}+s_{2} v_{1}=s_{1}+3 \text { and } s_{1} u_{2}+s_{2} v_{2}=s_{2}+3,
$$


respectively. Moreover, we can assume that $P_{1}$ and $P_{2}$ are coprime, and, without loss of generality, we can also assume that $s_{1} \leq s_{2}$. We consider different values of $s_{1}$.

Case $s_{1}=1$. If $s_{2}=1$, then in view of (2.1), we must have $u_{1}+v_{1}=4$ and $u_{2}+v_{2}=4$, that is, $\left(u_{i}, v_{i}\right)=(0,4),\left(u_{i}, v_{i}\right)=(1,3)$, $\left(u_{i}, v_{i}\right)=(2,2),\left(u_{i}, v_{i}\right)=(3,1)$ and $\left(u_{i}, v_{i}\right)=(4,0)$, for $i=1,2$.

If $s_{2}=2$, then, in view of (2.1), we must have $u_{1}+2 v_{1}=4$ and $u_{2}+2 v_{2}=5$, that is, $\left(u_{1}, v_{1}\right)=(0,2),\left(u_{1}, v_{1}\right)=(2,1)$ and $\left(u_{1}, v_{1}\right)=(4,0)$, while $\left(u_{2}, v_{2}\right)=(1,2),\left(u_{2}, v_{2}\right)=(3,1)$, and finally, $\left(u_{2}, v_{2}\right)=(5,0)$.

If $s_{2}=3$, then, in view of $(2.1)$, we must have $u_{1}+3 v_{1}=4$ and $u_{2}+3 v_{2}=6$, that is, $\left(u_{1}, v_{1}\right)=(1,1),\left(u_{1}, v_{1}\right)=(4,0)$, while $\left(u_{2}, v_{2}\right)=(0,2),\left(u_{2}, v_{2}\right)=(3,1)$, and finally, $\left(u_{2}, v_{2}\right)=(6,0)$.

If $s_{2}=4$, then, in view of $(2.1)$, we must have $u_{1}+4 v_{1}=4$ and $u_{2}+4 v_{2}=7$, that is, $\left(u_{1}, v_{1}\right)=(0,1),\left(u_{1}, v_{1}\right)=(4,0)$, while $\left(u_{2}, v_{2}\right)=(3,1)$, and finally, $\left(u_{2}, v_{2}\right)=(7,0)$.

If $s_{2}=4+l$ with $l \geq 1$, then equation (2.1) becomes

$$
u_{1}+(4+l) v_{1}=4 \text { and } u_{2}+(4+l) v_{2}=7+l .
$$

From the first equation of (2.2), we obtain $v_{1}=0$ and $u_{1}=4$. By the second equation of $(2.2)$, it follows that $v_{2} \in\{0,1\}$. If $v_{2}=0$, then $u_{2}=7+l$; if $v_{2}=1$, then $u_{2}=3$. In both cases, $P_{1}$ and $P_{2}$ are not coprime. Thus, this case is not considered.

Case $s_{1}=2$. Now, we have $s_{2} \geq 2$. If $s_{2}=2$, then in view of (2.1), we must have $2 u_{1}+2 v_{1}=5$ and $2 u_{2}+2 v_{2}=5$, which is not possible because 5 is not an even number.

If $s_{2}=3$, then, in view of (2.1), we must have $2 u_{1}+3 v_{1}=5$ and $2 u_{2}+3 v_{2}=6$, that is, $\left(u_{1}, v_{1}\right)=(1,1)$, while $\left(u_{2}, v_{2}\right)=(0,2)$ and $\left(u_{2}, v_{2}\right)=(3,0)$.

If $s_{2}=4$, then, in view of (2.1), we must have $2 u_{1}+4 v_{1}=5$ and $2 u_{2}+4 v_{2}=7$, which is not possible because 5 is not even.

If $s_{2}=5$, then, in view of (2.1), we must have $2 u_{1}+5 v_{1}=5$ and $2 u_{2}+5 v_{2}=8$, that is, $\left(u_{1}, v_{1}\right)=(0,1)$ and $\left(u_{2}, v_{2}\right)=(4,0)$.

If $s_{2}=5+l$ with $l \geq 1$, then equation (2.1) becomes

$$
2 u_{1}+(5+l) v_{1}=5 \text { and } 2 u_{2}+(5+l) v_{2}=8+l .
$$


The first equation of (2.3) is not possible because 5 is not an even number, $5+l \geq 6$ and $u_{1}$ and $v_{1}$ are non-negative integers.

Case $s_{1}=3$. Now, we have $s_{2} \geq 3$. If $s_{2}=3$, then in view of (2.1), we must have $3 u_{1}+3 v_{1}=6$ and $3 u_{2}+3 v_{2}=6$, that is, $\left(u_{i}, v_{i}\right)=(0,2)$, $\left(u_{i}, v_{i}\right)=(1,1)$ and $\left(u_{i}, v_{i}\right)=(2,0)$, for $i=1,2$.

If $s_{2}=3+l$ with $l \geq 1$, then in view of (2.1), we must have

$$
3 u_{1}+(3+l) v_{1}=6 \text { and } 3 u_{2}+(3+l) v_{2}=6+l .
$$

From the first equation of (2.4) we have that

$$
v_{1}=\frac{6-3 u_{1}}{3+l} \leq \frac{6}{3+l},
$$

and, using $l \geq 1$, then $v_{1} \in\{0,1\}$.

When $v_{1}=0$, then $3 u_{1}=6$; thus, $u_{1}=2$. Then, from the second equation of (2.4) we obtain that $v_{2} \in\{0,1\}$. If $v_{2}=0$, then $u_{2} \geq 1$, and, if $v_{2}=1$, then $u_{2}=1$. In both cases, we have that $P_{1}$ and $P_{2}$ are not coprime.

When $v_{1}=1$, then $3 u_{1}=3-l$, which is not possible since $u_{1}$ is an integer and $l \geq 1$.

Case $s_{1}=3+l$ with $l \geq 1$. Now, we have $s_{2} \geq 3+l$ with $l \geq 1$, and equation (2.1) becomes

$$
(3+l) u_{1}+s_{2} v_{1}=6+l=(3+l)+3
$$

and

$$
(3+l) u_{2}+s_{2} v_{2}=3+s_{2} .
$$

From the first equation of (2.5), and taking into account that $l \geq 1$, we obtain that $u_{1} \in\{0,1\}$.

When $u_{1}=0$, we must have $s_{2} v_{1}=6+l$, and since $s_{2} \geq 3+l$, we obtain

$$
v_{1}=\frac{6+l}{s_{2}} \leq \frac{(3+l)+3}{3+l}=1+\frac{3}{3+l} .
$$

Since $v_{1} \neq 0$ and $l \geq 1$, we must have $v_{1}=1$. Then $s_{2}=6+l$. Now, the second equation of (2.5) becomes

$$
(3+l) u_{2}+(6+l) v_{2}=(6+l)+3 .
$$


Then $v_{2}=0$. From equation $(2.6)$, we have $u_{2}=1+(6 / l+3)$. Thus, $l=3$ and $u_{2}=2$, and we obtain the systems with weight exponent $(6,9)$.

When $u_{1}=1$, we must have $s_{2} v_{1}=3$, and, since $s_{2} \geq 3+l$, we obtain $(3+l) v_{2} \leq 3$, which is not possible because $l \geq 1$. This concludes the proof of the proposition.

3. Weight exponent $\mathbf{s}=(1,1)$. A weight homogeneous polynomial system,

$$
\dot{x}=P_{1}(x, y) ; \quad \dot{y}=P_{2}(x, y),
$$

with weight exponent $(1,1)$ and weight degree $d$ is integrable, and its inverse integrating factor is $V(x, y)=x P_{2}(x, y)-y P_{1}(x, y)$. See [7] for more details.

As $P_{1}(x, y), P_{2}(x, y)$ and $V(x, y)$ are homogeneous polynomials, if the degree of $P_{1}(x, y)$ and $P_{2}(x, y)$ is $d$, then, of course, the degree of $V(x, y)$ is $d+1$. Thus, for $d=4$, we can write the homogeneous polynomials as follows:

$$
\begin{aligned}
P_{1}(x, y)= & \left(p_{1}-a_{1}\right) x^{4}+\left(p_{2}-4 a_{2}\right) x^{3} y+\left(p_{3}-6 a_{3}\right) x^{2} y^{2} \\
& +\left(p_{4}-4 a_{4}\right) x y^{3}-a_{5} y^{4} \\
P_{2}(x, y)= & a_{0} x^{4}+\left(4 a_{1}+p_{1}\right) x^{3} y+\left(6 a_{2}+p_{2}\right) x^{2} y^{2} \\
& +\left(4 a_{3}+p_{3}\right) x y^{3}+\left(a_{4}+p_{4}\right) y^{4},
\end{aligned}
$$

and

$$
V(x, y)=a_{0} x^{5}+5 a_{1} x^{4} y+10 a_{2} x^{3} y^{2}+10 a_{3} x^{2} y^{3}+5 a_{4} x y^{4}+a_{5} y^{5} .
$$

Thus, the first integral is

$$
H(x, y)=\int \frac{P_{1}(x, y)}{V(x, y)} d y+g(x),
$$

satisfying $\partial H / \partial x=-P_{2} / V$. The canonical forms appear in the factorization of $V$. Assume that $V(x, y)$ factorizes as:

(i) five simple real roots: $a_{0}\left(x-r_{1} y\right)\left(x-r_{2} y\right)\left(x-r_{3} y\right)\left(x-r_{4} y\right)(x-$ $\left.r_{5} y\right)$

(ii) one double and three simple real roots: $a_{0}\left(x-r_{1} y\right)^{2}\left(x-r_{2} y\right)(x-$ $\left.r_{3} y\right)\left(x-r_{4} y\right)$, 
(iii) two double roots and one simple real root: $a_{0}\left(x-r_{1} y\right)^{2}(x-$ $\left.r_{2} y\right)^{2}\left(x-r_{3} y\right)$

(iv) one triple and two simple real roots: $a_{0}\left(x-r_{1} y\right)^{3}\left(x-r_{2} y\right)\left(x-r_{3} y\right)$,

(v) one triple and one double real roots: $a_{0}\left(x-r_{1} y\right)^{3}\left(x-r_{2} y\right)^{2}$,

(vi) one quadruple and one simple real roots: $a_{0}\left(x-r_{1} y\right)^{4}\left(x-r_{2} y\right)$,

(vii) one quintuple real root: $a_{0}(x-r y)^{5}$,

(viii) three real and one couple of conjugate complex roots: $a_{0}(x-$ $\left.r_{1} y\right)\left(x-r_{2} y\right)\left(x-r_{3} y\right)\left(x^{2}+b x y+c y^{2}\right)$ with $b^{2}-4 c<0$,

(ix) one double, one simple real and one couple of conjugate complex roots: $a_{0}\left(x-r_{1} y\right)^{2}\left(x-r_{2} y\right)\left(x^{2}+b x y+c y^{2}\right)$ with $b^{2}-4 c<0$,

(x) one triple real and one couple of conjugate complex roots: $a_{0}(x-$ $\left.r_{1} y\right)^{3}\left(x^{2}+b x y+c y^{2}\right)$ with $b^{2}-4 c<0$,

(xi) one simple real and two couples of conjugate complex roots: $a_{0}(x-r y)\left(x^{2}+b_{1} x y+c_{1} y^{2}\right)\left(x^{2}+b_{2} x y+c_{2} y^{2}\right)$ with $b_{1}^{2}-4 c_{1}<$ $0, b_{2}^{2}-4 c_{2}<0$,

(xii) one simple real and one double couple of conjugate complex roots: $a_{0}(x-r y)\left(x^{2}+b x y+c y^{2}\right)^{2}$ with $b^{2}-4 c<0$.

Now, we shall compute the first integral for each case and obtain the conditions in order to show that it is a polynomial.

We define the function:

$$
f(r)=5\left(p_{4}+p_{3} r+p_{2} r^{2}+p_{1} r^{3}\right) .
$$

Case (i). A first integral $H$ is

$$
\left(x-r_{1} y\right)^{\gamma_{1}}\left(x-r_{2} y\right)^{\gamma_{2}}\left(x-r_{3} y\right)^{\gamma_{3}}\left(x-r_{4} y\right)^{\gamma_{4}}\left(x-r_{5} y\right)^{\gamma_{5}},
$$

where

$$
\gamma_{i}=\frac{f\left(r_{i}\right)+a_{0} \prod_{\substack{j=1 \\ j \neq i}}^{5}\left(r_{i}-r_{j}\right)}{\prod_{\substack{j=1 \\ j \neq i}}^{5}\left(r_{i}-r_{j}\right)} .
$$

We note that an integer power of $H$ is a polynomial if and only if $\gamma_{i} \in \mathbb{Q}$ for $i=1,2,3,4,5$, if they all have the same sign. 
Case (ii). A first integral $H$ is

$$
\begin{gathered}
\left(x-r_{1} y\right)^{\gamma_{1}}\left(x-r_{2} y\right)^{\gamma_{2}}\left(x-r_{3} y\right)^{\gamma_{3}}\left(x-r_{4} y\right)^{\gamma_{4}} \\
\exp \left(\frac{f\left(r_{1}\right) x}{r_{1}\left(r_{1}-r_{2}\right)\left(r_{1}-r_{3}\right)\left(r_{1}-r_{4}\right)\left(x-r_{1} y\right)}\right),
\end{gathered}
$$

with $\gamma_{1}=A_{1} / B_{1}=A_{1} /\left[\left(r_{1}-r_{2}\right)^{2}\left(r_{1}-r_{3}\right)^{2}\left(r_{1}-r_{4}\right)^{2}\right]$,

$$
\begin{aligned}
A_{1}= & 5 p_{1}\left(\left(r_{2}+r_{3}+r_{4}\right) r_{1}^{2}-2\left(r_{3} r_{4}+r_{2}\left(r_{3}+r_{4}\right)\right) r_{1}+3 r_{2} r_{3} r_{4}\right) r_{1}^{2} \\
& +5 p_{2}\left(r_{1}^{3}-\left(r_{3} r_{4}+r_{2}\left(r_{3}+r_{4}\right)\right) r_{1}+2 r_{2} r_{3} r_{4}\right) r_{1} \\
& +5 p_{3}\left(2 r_{1}^{3}-\left(r_{2}+r_{3}+r_{4}\right) r_{1}^{2}+r_{2} r_{3} r_{4}\right) \\
& +5 p_{4}\left(3 r_{1}^{2}-2\left(r_{2}+r_{3}+r_{4}\right) r_{1}+r_{3} r_{4}+r_{2}\left(r_{3}+r_{4}\right)\right)-2 a_{0} B_{1},
\end{aligned}
$$

for $i=2, \ldots, 4$ and

$$
\gamma_{i}=\frac{A_{i}}{B_{i}}=\frac{-\left(f\left(r_{i}\right)+a_{0} B_{i}\right)}{\left(r_{1}-r_{i}\right)^{2} \prod_{\substack{j=2 \\ j \neq i}}^{4}\left(r_{i}-r_{j}\right)} .
$$

We note that an integer power of $H$ is a polynomial if and only if $f\left(r_{1}\right)=0$ and $\gamma_{i} \in \mathbb{Q}$ for $i=1,2,3,4$, if they all have the same sign.

Case (iii). A first integral $H$ is

$$
\left(x-r_{1} y\right)^{\gamma_{1}}\left(x-r_{2} y\right)^{\gamma_{2}}\left(x-r_{3} y\right)^{\gamma_{3}} \exp \left(-\sum_{i=1}^{2} \frac{f\left(r_{i}\right) x}{r_{i}\left(r_{1}-r_{2}\right)^{2}\left(r_{i}-r_{3}\right)\left(r_{i} y-x\right)}\right),
$$

with $\gamma_{i}=A_{i} / B_{i}=A_{i} /\left[\left(r_{1}-r_{2}\right)^{3}\left(r_{i}-r_{3}\right)^{2}\right]$ for $i=1,2, \gamma_{3}=A_{3} / B_{3}=$ $-\left(f\left(r_{3}\right)+a_{0} B_{3}\right) /\left[\left(r_{1}-r_{3}\right)^{2}\left(r_{2}-r_{3}\right)^{2}\right]$,

$$
\begin{aligned}
A_{i}= & -2 a_{0} B_{i}+(-1)^{i+1}\left(5 p_{4}\left(3 r_{i}-r_{j}-2 r_{3}\right)-5 p_{3}\left(\left(r_{1}+r_{2}\right) r_{3}-2 r_{i}^{2}\right)\right. \\
& \left.+5 p_{2} r_{i}\left(r_{i}\left(r_{1}+r_{2}\right)-2 r_{j} r_{3}\right)+5 p_{1} r_{i}^{2}\left(-3 r_{j} r_{3}+r_{i}\left(2 r_{j}+r_{3}\right)\right)\right),
\end{aligned}
$$

for $i, j=1,2$ and $i \neq j$. We note that an integer power of $H$ is a polynomial if and only if $f\left(r_{i}\right)=0$ for $i=1,2$ and $\gamma_{i} \in \mathbb{Q}$ for $i=1,2,3$, if they all have the same sign.

Case (iv). A first integral $H$ is

$$
\left(x-r_{1} y\right)^{\gamma_{1}}\left(x-r_{2} y\right)^{\gamma_{2}}\left(x-r_{3} y\right)^{\gamma_{3}} \exp \left(\frac{5 \beta x}{2 r_{1}^{2}\left(r_{1}-r_{2}\right)^{2}\left(r_{1}-r_{3}\right)^{2}\left(r_{1} y-x\right)^{2}}\right),
$$


with

$$
\begin{aligned}
\beta= & \left(p_{1}\left(r_{1}^{2}-3 r_{2} r_{1}-3 r_{3} r_{1}+5 r_{2} r_{3}\right) r_{1}^{3}-p_{2}\left(r_{1}^{2}+r_{2} r_{1}+r_{3} r_{1}-3 r_{2} r_{3}\right) r_{1}^{2}\right. \\
& \left.-p_{3}\left(3 r_{1}^{2}-r_{2} r_{1}-r_{3} r_{1}-r_{2} r_{3}\right) r_{1}+p_{4}\left(-5 r_{1}^{2}+3 r_{2} r_{1}+3 r_{3} r_{1}-r_{2} r_{3}\right)\right) x \\
& +2 r_{1}\left(p_{1}\left(r_{1} r_{2}-2 r_{3} r_{2}+r_{1} r_{3}\right) r_{1}^{3}+p_{3}\left(2 r_{1}-r_{2}-r_{3}\right) r_{1}^{2}\right. \\
& \left.+p_{2}\left(r_{1}^{2}-r_{2} r_{3}\right) r_{1}^{2}+p_{4}\left(3 r_{1}^{2}-2 r_{2} r_{1}-2 r_{3} r_{1}+r_{2} r_{3}\right)\right) y, \\
\gamma_{1}= & A_{1} / B_{1}=A_{1} /\left[\left(r_{1}-r_{2}\right)^{3}\left(r_{1}-r_{3}\right)^{3}\right], \\
A_{1}= & -3 a_{0} B_{1}-5\left(r_{2}\left(p_{2}+p_{1} r_{2}\right) r_{1}^{3}+\left(r_{1}-3 r_{2}\right)\left(p_{2}+p_{1} r_{2}\right) r_{3} r_{1}^{2}\right. \\
& \left.+\left(p_{2} r_{2}^{2}+p_{1} r_{1}\left(r_{1}^{2}-3 r_{2} r_{1}+3 r_{2}^{2}\right)\right) r_{3}^{2}\right)+5 p_{3}\left(r_{1}^{3}-3 r_{2} r_{3} r_{1}\right. \\
& \left.+r_{2} r_{3}\left(r_{2}+r_{3}\right)\right)+5 p_{4}\left(3 r_{1}^{2}-3\left(r_{2}+r_{3}\right) r_{1}+r_{2}^{2}+r_{3}^{2}+r_{2} r_{3}\right), \\
\gamma_{i}= & A_{i} / B_{i}=(-1)^{i}\left(f\left(r_{i}\right)+a_{0} B_{i}\right) /\left[\left(r_{1}-r_{i}\right)^{3}\left(r_{2}-r_{3}\right)\right]
\end{aligned}
$$

for $i=2,3$. We note that an integer power of $H$ is a polynomial if and only if $\beta=0$ and $\gamma_{i} \in \mathbb{Q}$ for $i=1,2,3$, if they all have the same sign.

Case (v). A first integral $H$ is

$$
\left(x-r_{1} y\right)^{\gamma_{1}}\left(x-r_{2} y\right)^{\gamma_{2}} \exp (\beta),
$$

where

$$
\begin{aligned}
\beta= & \frac{2\left(r_{1}-r_{2}\right) x f\left(r_{2}\right) r_{1}^{2}}{r_{2}\left(r_{2} y-x\right)}+\frac{\left(r_{1}-r_{2}\right)^{2} x^{2} f\left(r_{1}\right)}{\left(x-r_{1} y\right)^{2}} \\
& +\frac{10\left(r_{1}-r_{2}\right)\left(\left(2 p_{3}+2 p_{1} r_{1} r_{2}+p_{2}\left(r_{1}+r_{2}\right)\right) r_{1}^{2}+p_{4}\left(3 r_{1}-r_{2}\right)\right) x}{r_{1} y-x}
\end{aligned}
$$

and

$$
\begin{aligned}
\gamma_{1}= & -2 r_{1}^{2}\left(3 a_{0}\left(r_{1}-r_{2}\right)^{4}+15 p_{4}+5 p_{3}\left(r_{1}+2 r_{2}\right)\right. \\
& \left.+5 r_{2}\left(3 p_{1} r_{1} r_{2}+p_{2}\left(2 r_{1}+r_{2}\right)\right)\right) \\
\gamma_{2}= & -2 r_{1}^{2}\left(2 a_{0}\left(r_{1}-r_{2}\right)^{4}-15 p_{4}-5 p_{3}\left(r_{1}+2 r_{2}\right)\right. \\
& \left.-5 r_{2}\left(3 p_{1} r_{1} r_{2}+p_{2}\left(2 r_{1}+r_{2}\right)\right)\right) .
\end{aligned}
$$

We note that an integer power of $H$ is a polynomial if and only if $\beta=0$ and $\gamma_{i} \in \mathbb{Q}$ for $i=1,2$, if they all have the same sign.

Case (vi). A first integral $H$ is

$$
\left(x-r_{1} y\right)^{\gamma_{1}}\left(x-r_{2} y\right)^{\gamma_{2}} \exp (\beta),
$$


where

$$
\begin{aligned}
\beta= & \frac{2\left(r_{1}-r_{2}\right)^{3} f\left(r_{1}\right) x^{3}}{r_{1}^{3}\left(r_{1} y-x\right)^{3}} \\
& +\frac{30\left(r_{1}-r_{2}\right)\left(\left(p_{3}+r_{2}\left(p_{2}+p_{1} r_{2}\right)\right) r_{1}^{3}+p_{4}\left(3 r_{1}^{2}-3 r_{2} r_{1}+r_{2}^{2}\right)\right) x}{r_{1}^{3}\left(r_{1} y-x\right)} \\
& +\frac{15\left(r_{1}-r_{2}\right)^{2}\left(p_{4}\left(3 r_{1}-2 r_{2}\right)+r_{1}\left(\left(p_{2}+p_{1} r_{2}\right) r_{1}^{2}+p_{3}\left(2 r_{1}-r_{2}\right)\right)\right) x^{2}}{r_{1}^{3}\left(x-r_{1} y\right)^{2}}
\end{aligned}
$$

and

$$
\begin{aligned}
& \gamma_{1}=-6\left(-4 a_{0}\left(r_{1}-r_{2}\right)^{4}+5 p_{4}+5 r_{2}\left(p_{3}+r_{2}\left(p_{2}+p_{1} r_{2}\right)\right)\right) \\
& \gamma_{2}=6\left(a_{0}\left(r_{1}-r_{2}\right)^{4}+5 p_{4}+5 r_{2}\left(p_{3}+r_{2}\left(p_{2}+p_{1} r_{2}\right)\right)\right) .
\end{aligned}
$$

We note that an integer power of $H$ is a polynomial if and only if $\beta=0$ and $\gamma_{i} \in \mathbb{Q}$ for $i=1,2$, if they all have the same sign.

Case (vii). A first integral $H$ is

$$
(x-r y)^{\gamma_{1}} \exp \left(\frac{\beta x}{(x-r y)^{4}}\right),
$$

where

$$
\begin{aligned}
\beta= & r x\left(r x\left(-p_{2} x+3 p_{1} r x+4 p_{2} r y\right)+p_{3}\left(x^{2}-4 r y x+6 r^{2} y^{2}\right)\right) \\
& -3 p_{4}(x-2 r y)\left(x^{2}-2 r y x+2 r^{2} y^{2}\right),
\end{aligned}
$$

and $\gamma_{1}=-12 a_{0} r^{4}$. We note that $x-r y$ is a polynomial first integral if and only if $\beta=0$.

Case (viii). A first integral $H$ is

$$
\begin{gathered}
\left(x-r_{1} y\right)^{\gamma_{1}}\left(x-r_{2} y\right)^{\gamma_{2}}\left(x-r_{3} y\right)^{\gamma_{3}}\left(x^{2}+b x y+c y^{2}\right)^{\gamma_{4}} \\
\exp \left(\frac{\beta x}{\prod_{i=1}^{3}\left(c+b r_{i}+r_{i}^{2}\right) \sqrt{\left(4 c-b^{2}\right) x^{2}}} \arctan \left(\frac{b x+2 c y}{\sqrt{\left(4 c-b^{2}\right) x^{2}}}\right)\right),
\end{gathered}
$$

where

$$
\begin{aligned}
\beta= & 5\left(2 p_{1} c^{3}-\left(b\left(p_{2}-p_{1}\left(r_{1}+r_{2}+r_{3}\right)\right)+2\left(p_{3}+p_{2}\left(r_{1}+r_{2}+r_{3}\right)\right.\right.\right. \\
& \left.\left.+p_{1}\left(r_{2} r_{3}+r_{1}\left(r_{2}+r_{3}\right)\right)\right)\right) c^{2}+\left(\left(p_{3}+p_{1} r_{1} r_{2}+p_{1}\left(r_{1}+r_{2}\right) r_{3}\right) b^{2}\right. \\
& +\left(3 p_{4}-3 p_{1} r_{1} r_{2} r_{3}+p_{3}\left(r_{1}+r_{2}+r_{3}\right)-p_{2}\left(r_{2} r_{3}+r_{1}\left(r_{2}+r_{3}\right)\right)\right) b \\
& +2\left(p_{2} r_{1} r_{2} r_{3}+p_{4}\left(r_{1}+r_{2}+r_{3}\right)+p_{3}\left(r_{2} r_{3}+r_{1}\left(r_{2}+r_{3}\right)\right)\right) c
\end{aligned}
$$


$-b p_{4}\left(b+r_{1}\right)\left(b+r_{2}\right)+\left(\left(p_{1} r_{1} b^{3}-p_{2} r_{1} b^{2}-p_{4} b+p_{3} r_{1} b-2 p_{4} r_{1}\right) r_{2}\right.$

$\left.\left.-b p_{4}\left(b+r_{1}\right)\right) r_{3}\right)$,

and $\gamma_{i}=A_{i} / B_{i}=-\left(f\left(r_{i}\right)+a_{0} B_{i}\right) /\left[\left(c+b r_{i}+r_{i}^{2}\right) \prod_{j=1, j \neq i}^{3}\left(r_{i}-r_{j}\right)\right]$ for $i=1,2,3, \gamma_{4}=A_{4} / B_{4}=A_{4} /\left[2 \prod_{i=1}^{3}\left(c+b r_{i}+r_{i}^{2}\right)\right]$

$$
\begin{aligned}
A_{4}= & -a_{0} B_{4}+5\left(\left(p_{2}+p_{1}\left(r_{1}+r_{2}+r_{3}\right)\right) c^{2}-\left(p_{4}+p_{1} r_{1} r_{2} r_{3}\right.\right. \\
& +p_{3}\left(r_{1}+r_{2}+r_{3}\right)+p_{2}\left(r_{2} r_{3}+r_{1}\left(r_{2}+r_{3}\right)\right) \\
& \left.+b\left(p_{3}-p_{1}\left(r_{2} r_{3}+r_{1}\left(r_{2}+r_{3}\right)\right)\right)\right) c+p_{4}\left(b+r_{1}\right)\left(b+r_{2}\right) \\
& \left.+\left(p_{4}\left(b+r_{1}\right)+\left(p_{4}+\left(p_{1} b^{2}-p_{2} b+p_{3}\right) r_{1}\right) r_{2}\right) r_{3}\right) .
\end{aligned}
$$

We note that an integer power of $H$ is a polynomial if and only if $\beta=0$ and $\gamma_{i} \in \mathbb{Q}$ for $i=1,2,3,4$, if they all have the same sign.

Case (ix). A first integral $H$ is

$$
\begin{aligned}
& \left(x-r_{1} y\right)^{\gamma_{1}}\left(x-r_{2} y\right)^{\gamma_{2}}\left(x^{2}+b x y+c y^{2}\right)^{\gamma_{3}} \\
& \exp \left(-\frac{f\left(r_{1}\right) x}{r_{1}\left(r_{1}-r_{2}\right)\left(c+r_{1} b+r_{1}^{2}\right)\left(r_{1} y-x\right)}\right. \\
& \left.\quad+\frac{\beta x}{\prod_{i=1}^{2}\left(c+b r_{i}+r_{i}^{2}\right)^{3-i} \sqrt{\left(4 c-b^{2}\right) x^{2}}} \arctan \left(\frac{b x+2 c y}{\sqrt{\left(4 c-b^{2}\right) x^{2}}}\right)\right),
\end{aligned}
$$

where

$$
\begin{aligned}
\beta= & 5\left(2 p_{1} c^{3}-\left(b\left(p_{2}-p_{1}\left(2 r_{1}+r_{2}\right)\right)+2\left(p_{3}+p_{2}\left(2 r_{1}+r_{2}\right)\right.\right.\right. \\
& \left.\left.+p_{1} r_{1}\left(r_{1}+2 r_{2}\right)\right)\right) c^{2}+\left(\left(p_{3}+p_{1} r_{1}\left(r_{1}+2 r_{2}\right)\right) b^{2}\right. \\
& +\left(3 p_{4}+p_{3}\left(2 r_{1}+r_{2}\right)-r_{1}\left(3 p_{1} r_{1} r_{2}+p_{2}\left(r_{1}+2 r_{2}\right)\right)\right) b \\
& \left.+2\left(p_{4}\left(2 r_{1}+r_{2}\right)+r_{1}\left(p_{2} r_{1} r_{2}+p_{3}\left(r_{1}+2 r_{2}\right)\right)\right)\right) c-b p_{4}\left(b+r_{1}\right)^{2} \\
& \left.+\left(-p_{4} b^{2}-2 p_{4} r_{1} b+\left(b\left(p_{1} b^{2}-p_{2} b+p_{3}\right)-2 p_{4}\right) r_{1}^{2}\right) r_{2}\right), \\
\gamma_{1}= & -\frac{A_{1}}{B_{1}}=-\frac{A_{1}}{\left(r_{1}-r_{2}\right)^{2}\left(c+b r_{1}+r_{1}^{2}\right)^{2}}, \\
A_{1}= & 2 a_{0} c^{2}\left(r_{1}-r_{2}\right)^{2}+2 a_{0} b^{2} r_{1}^{2}\left(r_{1}-r_{2}\right)^{2} \\
& +c\left(-5 p_{4}+r_{1}\left(4 a_{0}\left(b+r_{1}\right)\left(r_{1}-r_{2}\right)^{2}+5 p_{1} r_{1}\left(2 r_{1}-3 r_{2}\right)\right.\right. \\
& \left.\left.+5 p_{2}\left(r_{1}-2 r_{2}\right)\right)-5 p_{3} r_{2}\right)+b\left(\left(4 a_{0} r_{1}\left(r_{1}-r_{2}\right)^{2}-5 p_{3}\right.\right. \\
& \left.\left.+5 p_{1} r_{1}\left(r_{1}-2 r_{2}\right)-5 p_{2} r_{2}\right) r_{1}^{2}+5 p_{4}\left(r_{2}-2 r_{1}\right)\right)+r_{1}\left(5 p_{4}\left(2 r_{2}-3 r_{1}\right)\right. \\
& \left.+r_{1}\left(\left(2 a_{0}\left(r_{1}-r_{2}\right)^{2}-5 p_{2}-5 p_{1} r_{2}\right) r_{1}^{2}+5 p_{3}\left(r_{2}-2 r_{1}\right)\right)\right),
\end{aligned}
$$




$$
\begin{aligned}
\gamma_{2}= & \frac{A_{2}}{B_{2}}=\frac{-f\left(r_{2}\right)+a_{0} B_{2}}{\left(r_{1}-r_{2}\right)^{2}\left(c+b r_{2}+r_{2}^{2}\right)}, \\
\gamma_{3}= & \frac{A_{3}}{B_{3}}=\frac{A_{3}}{2\left(c+b r_{1}+r_{1}^{2}\right)^{2}\left(c+b r_{2}+r_{2}^{2}\right)}, \\
A_{3}= & -a_{0} B_{3}+5\left(\left(p_{2}+p_{1}\left(2 r_{1}+r_{2}\right)\right) c^{2}-\left(p_{4}+p_{3}\left(2 r_{1}+r_{2}\right)\right.\right. \\
& \left.+r_{1}\left(p_{1} r_{1} r_{2}+p_{2}\left(r_{1}+2 r_{2}\right)\right)+b\left(p_{3}-p_{1} r_{1}\left(r_{1}+2 r_{2}\right)\right)\right) c \\
& \left.+p_{4}\left(b+r_{1}\right)^{2}+\left(\left(p_{1} b^{2}-p_{2} b+p_{3}\right) r_{1}^{2}+2 p_{4} r_{1}+b p_{4}\right) r_{2}\right) .
\end{aligned}
$$

We note that an integer power of $H$ is a polynomial if and only if $f\left(r_{1}\right)=0, \beta=0$ and $\gamma_{i} \in \mathbb{Q}$ for $i=1,2,3$, if they all have the same sign.

Case (x). A first integral $H$ is

$$
\begin{aligned}
& \left(x-r_{1} y\right)^{\gamma_{1}}\left(x^{2}+b x y+c y^{2}\right)^{\gamma_{2}} \exp \left(\frac{f\left(r_{1}\right)\left(c+b r_{1}+r_{1}^{2}\right)^{2} x^{2}}{r_{1}^{2}\left(x-r_{1} y\right)^{2}}\right. \\
& \left.-\frac{\beta_{1}\left(c+b r_{1}+r_{1}^{2}\right) x}{r_{1}^{2}\left(x-r_{1} y\right)}+\frac{\beta_{2} x}{\sqrt{\left(4 c-b^{2}\right) x^{2}}} \arctan \left(\frac{b x+2 c y}{\sqrt{\left(4 c-b^{2}\right) x^{2}}}\right)\right)
\end{aligned}
$$

where

$$
\begin{aligned}
\beta_{1}= & 10\left(\left(p_{2}-b p_{1}\right) r_{1}^{4}+2\left(p_{3}-c p_{1}\right) r_{1}^{3}+\left(-c p_{2}+b p_{3}+3 p_{4}\right) r_{1}^{2}\right. \\
& \left.+2 b p_{4} r_{1}+c p_{4}\right), \\
\beta_{2}= & 10\left(\left(p_{1} r_{1}^{3}-p_{4}\right) b^{3}-r_{1}\left(p_{2} r_{1}^{2}+3 p_{4}\right) b^{2}+r_{1}^{2}\left(p_{3} r_{1}-3 p_{4}\right) b\right. \\
& -2 p_{4} r_{1}^{3}+2 c^{3} p_{1}-c^{2}\left(2 p_{3}+b\left(p_{2}-3 p_{1} r_{1}\right)+6 r_{1}\left(p_{2}+p_{1} r_{1}\right)\right) \\
& +c\left(\left(3 p_{1} r_{1}^{2}+p_{3}\right) b^{2}+3\left(p_{4}+r_{1}\left(p_{3}-r_{1}\left(p_{2}+p_{1} r_{1}\right)\right)\right) b\right. \\
& \left.\left.+2 r_{1}\left(3 p_{4}+r_{1}\left(3 p_{3}+p_{2} r_{1}\right)\right)\right)\right),
\end{aligned}
$$

and

$$
\begin{aligned}
\gamma_{1}= & -2\left(3 a_{0}\left(c+r_{1}\left(b+r_{1}\right)\right)^{3}+5\left(\left(p_{1} b^{2}-p_{2} b+p_{3}\right) r_{1}^{3}+3 p_{4} r_{1}^{2}\right.\right. \\
& +3 b p_{4} r_{1}+b^{2} p_{4}+c^{2}\left(p_{2}+3 p_{1} r_{1}\right)-c\left(p_{4}+b\left(p_{3}-3 p_{1} r_{1}^{2}\right)\right. \\
& \left.\left.\left.+r_{1}\left(3 p_{3}+r_{1}\left(3 p_{2}+p_{1} r_{1}\right)\right)\right)\right)\right) \\
\gamma_{2}= & 5\left(\left(p_{1} b^{2}-p_{2} b+p_{3}\right) r_{1}^{3}+3 p_{4} r_{1}^{2}+3 b p_{4} r_{1}+b^{2} p_{4}+c^{2}\left(p_{2}+3 p_{1} r_{1}\right)\right. \\
& \left.-c\left(p_{4}+b\left(p_{3}-3 p_{1} r_{1}^{2}\right)+r_{1}\left(3 p_{3}+r_{1}\left(3 p_{2}+p_{1} r_{1}\right)\right)\right)\right) \\
& -2 a_{0}\left(c+r_{1}\left(b+r_{1}\right)\right)^{3} .
\end{aligned}
$$


We note that an integer power $H$ is a polynomial if and only if $f\left(r_{1}\right)=0, \beta_{1}=\beta_{2}=0$ and $\gamma_{i} \in \mathbb{Q}$ for $i=1,2$, if they all have the same sign.

Case (xi). A first integral $H$ is

$$
\begin{aligned}
& \left(x^{2}+b_{1} x y+c_{1} y^{2}\right)^{\gamma_{1}}\left(x^{2}+b_{2} x y+c_{2} y^{2}\right)^{\gamma_{2}}\left(x-r_{1} y\right)^{\gamma_{3}} \\
& \exp \left(\sum_{i=1}^{2} \frac{\beta_{i} x}{\sqrt{\left(4 c_{i}-b_{i}^{2}\right) x^{2}}} \arctan \left(\frac{b_{i} x+2 c_{i} y}{\sqrt{\left(4 c_{i}-b_{i}^{2}\right) x^{2}}}\right)\right),
\end{aligned}
$$

with $\beta_{i}=\alpha_{i} / \delta_{i}$ for $i=1,2$, where

$$
\begin{aligned}
\alpha_{i}= & 5\left(-b_{i}\left(c_{i}^{2} p_{2}+c_{i} c_{j} p_{2}+b_{j} c_{i}\left(c_{i} p_{1}+p_{3}\right)-3 c_{i} p_{4}+c_{j} p_{4}\right)\right. \\
& +b_{i}\left(c_{i}^{2} p_{1}+c_{j} p_{3}+c_{i}\left(-3 c_{j} p_{1}+b_{j} p_{2}+p_{3}\right)+b_{j} p_{4}\right) r_{1} \\
& +b_{i}^{3}\left(-p_{4}+c_{j} p_{1} r_{1}\right)+b_{i}^{2}\left(c_{i} c_{j} p_{1}+c_{i} p_{3}+b_{j} p_{4}-\left(b_{j} c_{i} p_{1}+c_{j} p_{2}+p_{4}\right) r_{1}\right) \\
& +2\left(c_{i}^{3} p_{1}-c_{j} p_{4} r_{1}-c_{i}^{2}\left(c_{j} p_{1}+p_{3}+p_{2} r_{1}-b_{j}\left(p_{2}+p_{1} r_{1}\right)\right)\right. \\
& \left.\left.+c_{i}\left(p_{4} r_{1}+c_{j}\left(p_{3}+p_{2} r_{1}\right)-b_{j}\left(p_{4}+p_{3} r_{1}\right)\right)\right)\right), \\
\delta_{i}= & \left(\left(b_{2}^{2} c_{1}+\left(c_{1}-c_{2}\right)^{2}+b_{1}^{2} c_{2}-b_{1} b_{2}\left(c_{1}+c_{2}\right)\right)\left(c_{i}+b_{i} r_{1}+r_{1}^{2}\right),\right.
\end{aligned}
$$

for $i, j=1,2$ and $i \neq j$,

$$
\begin{aligned}
\gamma_{i}= & -2 a_{0}\left(b_{j}^{2} c_{i}+\left(c_{i}-c_{j}\right)^{2}+b_{i}^{2} c_{j}-b_{i} b_{j}\left(c_{i}+c_{j}\right)\right)\left(c_{i}+r_{1}\left(b_{i}+r_{1}\right)\right) \\
& +5\left(b_{i}^{2}\left(p_{4}+c_{j} p_{1} r_{1}\right)+b_{i}\left(c_{i} c_{j} p_{1}-c_{i} p_{3}-c_{j} p_{2} r_{1}+p_{4} r_{1}\right)\right. \\
& +\left(c_{i}-c_{j}\right)\left(-p_{4}-p_{3} r_{1}+c_{i}\left(p_{2}+p_{1} r_{1}\right)\right)-b_{j}\left(c_{i}^{2} p_{1}+p_{4}\left(b_{i}+r_{1}\right)\right. \\
& \left.\left.-c_{i}\left(p_{3}+\left(-b_{i} p_{1}+p_{2}\right) r_{1}\right)\right)\right),
\end{aligned}
$$

for $i, j=1,2$ and $i \neq j$. Finally,

$$
\gamma_{3}=\frac{A_{3}}{B_{3}}=\frac{-\left(f\left(r_{1}\right)+a_{0} B_{3}\right)}{\prod_{i=1}^{2}\left(c_{i}+b_{i} r_{1}+r_{1}^{2}\right)} .
$$

We note that an integer power of $H$ is a polynomial if and only if $\beta_{1}=\beta_{2}=0$ and $\gamma_{i} \in \mathbb{Q}$ for $i=1,2,3$, if they all have the same sign.

Case (xii). A first integral $H$ is

$$
(x-r y)^{\gamma_{1}}\left(x^{2}+b x y+c y^{2}\right)^{\gamma_{2}} \exp \left(\frac{\beta_{1} x^{3}}{\left[\left(4 c-b^{2}\right) x^{2}\right]^{3 / 2}} \arctan \left(\frac{b x+2 c y}{\sqrt{\left(4 c-b^{2}\right) x^{2}}}\right)\right.
$$




$$
\left.+\frac{10 \beta_{2}\left(c+b r+r^{2}\right) x}{\left(b^{2}-4 c\right) c\left(x^{2}+b x y+c y^{2}\right)}\right)
$$

where

$$
\begin{aligned}
\beta_{1}= & -10\left(\left(p_{4}+r\left(p_{3}-r\left(p_{2}+p_{1} r\right)\right)\right) b^{3}+4 p_{3} r^{2} b^{2}+2 r^{2}\left(p_{3} r-3 p_{4}\right) b\right. \\
& -4 p_{4} r^{3}+4 c^{3} p_{1}+c^{2}\left(4\left(p_{3}+r\left(p_{2}+3 p_{1} r\right)\right)-2 b\left(p_{2}-3 p_{1} r\right)\right) \\
& -2 c\left(2 p_{2} r b^{2}+\left(3 p_{4}-r\left(p_{3}+r\left(3 p_{1} r-p_{2}\right)\right)\right) b\right. \\
& \left.\left.+2 r\left(3 p_{4}+r\left(p_{3}+p_{2} r\right)\right)\right)\right), \\
\beta_{2}= & -p_{4} y b^{3}+\left(c\left(p_{1} r x+p_{3} y\right)-p_{4}(x+r y)\right) b^{2}+\left(\left(p_{1}(x+r y)-p_{2} y\right) c^{2}\right. \\
& \left.+\left(-p_{2} r x+3 p_{4} y+p_{3}(x+r y)\right) c-p_{4} r x\right) b \\
& +2 c\left(p_{1} y c^{2}-\left(p_{1} r x+p_{3} y+p_{2}(x+r y)\right) c+p_{3} r x+p_{4}(x+r y)\right), \\
\gamma_{1}= & 2\left(a_{0}\left(c+b r+r^{2}\right)^{2}+f(r)\right), \\
\gamma_{2}= & 4 a_{0}\left(c+b r+r^{2}\right)^{2}-f(r) .
\end{aligned}
$$

We note that an integer power of $H$ is a polynomial if and only if $\beta_{1}=\beta_{2}=0$ and $\gamma_{i} \in \mathbb{Q}$ for $i=1,2$, if they all have the same sign.

4. Weight exponent $\mathbf{s}=(1,2)$. In this section, we prove Theorem 1.3. Since systems in equation (1.5) are homogeneous, we know that they are integrable because they have the inverse integrating factor $V=x \dot{y}-y \dot{x}$. The strategy will be to obtain such first integrals and to determine which of them are polynomials. Denoting systems in equation (1.5) by $\dot{x}=P(x, y)$ and $\dot{y}=Q(x, y)$, the first integral is

$$
H(x, y)=\int \frac{P(x, y)}{V(x, y)} d y+g(x)
$$

satisfying $\partial H / \partial x=-Q(x, y) / V(x, y)$.

The first system in equation (1.5) has the first integral

$$
H=x^{-3-2 p_{2}}\left(3 y^{2}-x^{2}\right)^{-3+p_{2}} \exp \left(-2 \sqrt{3} p_{1} \operatorname{arctanh}\left(\frac{x}{\sqrt{3} y}\right)\right) .
$$

Note that an integer power of $H$ is a polynomial if and only if $p_{1}=0$ and $p_{2}=3(1-q) /(1+2 q)$ with $q \in \mathbb{Q}^{+}$. 
The second system in equation (1.5) has the first integral

$$
H=x^{-3-2 p_{2}}\left(3 y^{2}+x^{2}\right)^{-3+p_{2}} \exp \left(-2 \sqrt{3} p_{1} \arctan \left(\frac{x}{\sqrt{3} y}\right)\right) .
$$

Note that an integer power of $H$ is a polynomial if and only if $p_{1}=0$ and $p_{2}=3(1-q) /(1+2 q)$ with $q \in \mathbb{Q}^{+}$.

The third system in equation (1.5) has the first integral

$$
H=x^{-2\left(3+p_{1}\right)} y^{-3+2 p_{1}} \exp \frac{2 p_{2} y}{x} .
$$

Note that an integer power of $H$ is a polynomial if and only if $p_{2}=0$ and $p_{1}=3(1-2 q) /(2(1+q))$ with $q \in \mathbb{Q}^{+}$.

The fourth system in equation (1.5) has the first integral

$$
H=x \exp \left(-y \frac{2 p_{1} x+p_{2} y}{3 x^{2}}\right) .
$$

Note that an integer power of $H$ is a polynomial if and only if $p_{1}=$ $p_{2}=0$.

The fifth system in equation (1.5) has the first integral $x / y$ which is never a polynomial.

5. Weight exponent $\mathbf{s}=(1,3)$. Performing a change of variables $(X, Y)=\left(x^{3}, y\right)$, the planar weight homogeneous systems of weight degree 4 and weight exponent $(1,3)$ become

$$
\dot{X}=3 a_{40} X^{2}+3 a_{11} X Y, \quad \dot{Y}=b_{60} X^{2}+b_{31} X Y+b_{02} Y^{2} .
$$

Again, we shall use the inverse integrating factor $V=X \dot{Y}-Y \dot{X}$ for computing the first integrals of system (5.1).

It is clear that, if $a_{11}=a_{40}=0$, then a polynomial first integral is $X$, and, if $b_{60}=b_{31}=b_{02}=0$, then a polynomial first integral is $Y$.

Now, we consider the other cases.

Case (i). $3 a_{11}-b_{02} \neq 0$ and $R=-\left(3 a_{40}-b_{31}\right)^{2}+4\left(-3 a_{11}+\right.$ $\left.b_{02}\right) b_{60} \neq 0$. In this case, system (5.1) has the first integral

$$
\frac{6}{\sqrt{R}}\left(a_{11}\left(3 a_{40}+b_{31}\right)-2 a_{40} b_{02}\right) \arctan \left(\frac{3 a_{40} X-b_{31} X+6 a_{11} Y-2 b_{02} Y}{\sqrt{R} X}\right)
$$


$+2\left(3 a_{11}-b_{02}\right) \log X+3 a_{11} \log \left(\frac{Y\left(3 a_{40} X-b_{31} X+3 a_{11} Y-b_{02} Y\right)}{X^{2}}-b_{60}\right)$.

Here, $\log A$ always means $\log |A|$ and, as usual, log is the logarithm in base $e$. Since this first integral must be a polynomial, we must have

$$
a_{11}\left(3 a_{40}+b_{31}\right)-2 a_{40} b_{02}=0 .
$$

Now, we consider different subcases.

If $3 a_{11}-2 b_{02} \neq 0$, then from equation (5.2), we obtain

$$
a_{40}=-\frac{a_{11} b_{31}}{3 a_{11}-2 b_{02}} .
$$

Therefore, using the exponential of the previous first integral, we obtain that the first integral is

$$
H=X^{1-\left(3 a_{11} / 6 a_{11}-2 b_{02}\right)} p(X, Y)^{3 a_{11} /\left(6 a_{11}-2 b_{02}\right)},
$$

where

$$
\begin{aligned}
p(X, Y)= & \left(3 a_{11}-2 b_{02}\right) b_{60} X^{2}+2\left(3 a_{11}-b_{02}\right) b_{31} X Y \\
& -\left(9 a_{11}^{2}-9 a_{11} b_{02}+2 b_{02}^{2}\right) Y^{2}
\end{aligned}
$$

Note that, since $a_{11}-b_{02} \neq 0$ and $3 a_{11}-2 b_{02} \neq 0$, we have $9 a_{11}^{2}-$ $9 a_{11} b_{02}+2 b_{02}^{2} \neq 0$. Therefore, an integer power of $H$ is a polynomial first integral if and only if $3 a_{11} /\left(6 a_{11}-2 b_{02}\right)=m / n \in \mathbb{Q}^{+}$, and $m / n<1$. In this case, the first integral $H$ is

$$
\begin{aligned}
X^{n-m}\left(\left(3 a_{11}-2 b_{02}\right) b_{60} X^{2}\right. & +2\left(3 a_{11}-b_{02}\right) b_{31} X Y \\
& \left.-\left(9 a_{11}^{2}-9 a_{11} b_{02}+2 b_{02}^{2}\right) Y^{2}\right)^{m}
\end{aligned}
$$

If $3 a_{11}-2 b_{02}=0$, that is, $b_{02}=3 a_{11} / 2$. In this case, from equation (5.2), we obtain $a_{11} b_{31}=0$. Hence, either $a_{11}=0$ or $b_{31}=0$. However, if $a_{11}=0$, then $b_{02}=0$, which contradicts the fact that $3 a_{11}-2 b_{02} \neq 0$. Therefore, this case is not possible and we must have $b_{31}=0$. Then, the first integral is

$$
H=\frac{-2 b_{60} X^{2}+6 a_{40} X Y+3 a_{11} Y^{2}}{X},
$$

which is never a polynomial. 
Case (ii). $b_{02}=3 a_{11}$ and $b_{31}-3 a_{40} \neq 0$. In this case, system (5.1) has the first integral

$$
\begin{aligned}
\left(b_{31}\right. & \left.-3 a_{40}\right)^{2} \log X+\frac{3 a_{11}\left(3 a_{40}-b_{31}\right) Y}{X} \\
& +3\left(-3 a_{40}^{2}+b_{31} a_{40}-a_{11} b_{60}\right) \log \left(-\frac{b_{60} X-3 a_{40} Y+b_{31} Y}{X}\right) .
\end{aligned}
$$

In order for the first integral to be a polynomial, we must have $a_{11}\left(3 a_{40}-b_{31}\right)=0$, that is, $a_{11}=0$ (and hence, $\left.b_{02}=0\right)$. Then, using the exponential of the previous first integral, we obtain the following first integral

$$
X^{1 / 3}\left(\frac{b_{60} X-3 a_{40} Y+b_{31} Y}{X}\right)^{a_{40} /\left(3 a_{40}-b_{31}\right)} .
$$

Then, we must have $b_{31} / a_{40}=-m / n$ with $m / n \in \mathbb{Q}^{+}$. In this case, the previous first integral becomes $H=X^{m}\left(b_{60} X-3 a_{40} Y+b_{31} Y\right)^{3 n}$.

Case (iii). $b_{02}=3 a_{11}$ and $b_{31}=3 a_{40}$. System (5.1) has the first integral

$$
\frac{-2 b_{60} X^{2} \log X+6 a_{40} Y X+3 a_{11} Y^{2}}{6 X^{2}} .
$$

Since the case $a_{40}=a_{11}=0$ has been studied, we have that in this case the first integral is never a polynomial.

Case (iv). $3 a_{11}-b_{02} \neq 0$ and $R=0$. Then,

$$
b_{06}=\frac{-\left(3 a_{40}-b_{31}\right)^{2}}{4\left(3 a_{11}-b_{02}\right)},
$$

and system (5.1) has the first integral

$$
\begin{aligned}
& \frac{3\left(-3 a_{11} a_{40}+2 b_{02} a_{40}-a_{11} b_{31}\right) X}{3 a_{40} X-b_{31} X+6 a_{11} Y-2 b_{02} Y}+\left(3 a_{11}-b_{02}\right) \log \left(36 a_{11} X-12 b_{02} X\right) \\
& +3 a_{11} \log \left(-\frac{3 a_{40} X-b_{31} X+6 a_{11} Y-2 b_{02} Y}{X}\right) .
\end{aligned}
$$

In order to show that it is a polynomial, we must have

$$
2 a_{40} b_{02}-a_{11}\left(3 a_{40}+b_{31}\right)=0 .
$$

We will now consider two different subcases. 
If $3 a_{11} \neq 2 b_{02}$, then condition (5.3) becomes

$$
a_{40}=-\frac{a_{11} b_{31}}{3 a_{11}-2 b_{02}} .
$$

Using the exponential of the previous first integral, we obtain the following first integral:

$$
X^{1 /\left(36 a_{11}-12 b_{02}\right)}\left(\frac{b_{31} X-3 a_{11} Y+2 b_{02} Y}{X}\right)^{a_{11} / 4\left(b_{02}-3 a_{11}\right)^{2}}
$$

From this first integral, we obtain the first integral:

$$
X\left(b_{31} X-3 a_{11} Y+2 b_{02} Y\right)^{-3 a_{11} / b_{02}} .
$$

So, if $b_{02} \neq 0$, then $3 a_{11} / b_{02}=-m / n$ with $m / n \in \mathbb{Q}^{+}$, and the polynomial first integral is

$$
X^{n}\left(b_{31} X-3 a_{11} Y+2 b_{02} Y\right)^{m} .
$$

If $b_{02}=0$, then $H=b_{31} X-3 a_{11} Y$ is a polynomial first integral. This concludes the proof of Theorem 1.4.

6. Weight exponent $\mathbf{s}=(1,4)$. We introduce the change $(X, Y)=$ $\left(x^{4}, y\right)$ in the planar weight homogeneous polynomial differential systems (1.1) of weight degree 4 with weight exponent $(1,4)$. In these new variables $(X, Y)$, the systems with weight exponent $(1,4)$ become, after introducing the new independent variable $d \tau=x^{3} d t$, as follows:

$$
X^{\prime}=4\left(a_{40} X+a_{01} Y\right), \quad Y^{\prime}=b_{70} X+b_{31} Y,
$$

where the prime denotes the derivative with respect to $\tau$. If $a_{40}=$ $a_{01}=0$, then a polynomial first integral is $X$, and, if $b_{70}=b_{31}=0$, then a polynomial first integral is $Y$.

Now, we consider the other cases.

Case (i). $R=-\left(4 a_{40}-b_{31}\right)^{2}-16 a_{01} b_{70} \neq 0$. Now, a first integral of system (6.1) is:

$$
\begin{gathered}
\frac{2\left(4 a_{40}+b_{31}\right)}{\sqrt{R}} \arctan \left(\frac{\left(4 a_{40}-b_{31}\right) X+8 a_{01} Y}{\sqrt{R} X}\right) \\
+\log \left(-b_{70} X^{2}+\left(4 a_{40}-b_{31}\right) X Y+4 a_{01} Y^{2}\right) .
\end{gathered}
$$


Since it must be a polynomial, we must have $b_{31}=-4 a_{40}$ such that the polynomial first integral is $H=-b_{70} X^{2}+8 a_{40} X Y+4 a_{01} Y^{2}$.

Case (ii). $R=0$. We consider different subcases.

First, we study when $b_{70} \neq 0$. Then, from $R=0$, we obtain

$$
a_{01}=-\frac{\left(4 a_{40}-b_{31}\right)^{2}}{16 b_{70}} .
$$

If $b_{31}-4 a_{40} \neq 0$, then the first integral is:

$$
\frac{2\left(4 a_{40}+b_{31}\right) b_{70} X}{-2 b_{70} X+\left(4 a_{40}-b_{31}\right) Y}+\left(4 a_{40}-b_{31}\right) \log \left(2 b_{70} X+\left(-4 a_{40}+b_{31}\right) Y\right),
$$

which is a polynomial if and only if $b_{31}=-4 a_{40}$. The polynomial first integral is $b_{70} X-4 a_{40} Y$.

If $b_{31}=4 a_{40}$, then $a_{40} \neq 0$ (otherwise, $b_{31}=0$ and, from equation (6.2), we also have $a_{01}=0$, which has already been considered), and the first integral of equation (6.1) is

$$
H=\frac{Y}{X}-\frac{b_{70} \log X}{4 a_{40}},
$$

which is never a polynomial.

If $b_{70}=0$, then from $R=0$, we obtain $b_{31}=4 a_{40}$. We only consider the case $a_{40} \neq 0$, where the first integral of equation (6.1) is:

$$
-\frac{a_{40} X}{Y}+a_{01} \log Y
$$

which is never a polynomial. This completes the proof of Theorem 1.5.

7. Weight exponent $s=(2,3)$. We prove Theorem 1.6. The planar weight homogeneous polynomial differential systems (1.1) with weight degree 4 and weight-exponent $(2,3)$ are:

$$
\dot{x}=a_{11} x y, \quad \dot{y}=b_{30} x^{3}+b_{02} y^{2} .
$$

If $a_{11}\left(3 a_{11}-2 b_{02}\right) \neq 0$, then the first integral of system (7.1) is:

$$
H=x^{-2 b_{02} / a_{11}}\left(2 b_{30} x^{3}-3 a_{11} y^{2}+2 b_{02} y^{2}\right) .
$$


Then, an integer power of $H$ is a polynomial first integral if and only if $-2 b_{02} / a_{11} \in \mathbb{Q}^{+}$.

If $a_{11}=0$, then $H=x$ is a polynomial first integral of system (7.1). If $b_{30}=b_{02}=0$, then $H=y$ is a polynomial first integral of system (7.1). If $a_{11} \neq 0$ and $3 a_{11}=2 b_{02}$, then the first integral of system (7.1) is:

$$
H=\frac{y^{4}}{x^{3}}-\frac{2 b_{30}}{a_{11}} \log x,
$$

which is never a polynomial. This completes the proof of Theorem 1.6.

8. Weight exponent $\mathbf{s}=(2,5)$. Here, we prove Theorem 1.7. The planar weight homogeneous polynomial differential systems (1.1) of weight degree 4 with weight exponent $(2,5)$ are:

$$
\dot{x}=a_{01} y, \quad \dot{y}=b_{40} x^{4} .
$$

It is straightforward to prove that $H=2 b_{40} x^{5}-5 a_{01} y^{2}$ is a polynomial first integral.

9. Weight exponent $\mathbf{s}=(6,9)$. Now, we prove Theorem 1.8. The planar weight homogeneous polynomial differential systems (1.1) of weight degree 4 with weight exponent $(6,9)$ are:

$$
\dot{x}=a_{01} y, \quad \dot{y}=b_{20} x^{2} .
$$

It is straightforward to prove that $H=2 b_{20} x^{3}-3 a_{01} y^{2}$ is a polynomial first integral. This completes the proof of Theorem 1.8.

\section{REFERENCES}

1. A. Algaba, E. Freire, E. Gamero and C. García, Monodromy, center-focus and integrability problems for quasi-homogeneous polynomial systems, Nonlin. Anal. 72 (2010), 1726-1736.

2. A. Algaba, N. Fuentes and C. García, Centers of quasi-homogeneous polynomial planar systems, Nonlin. Anal. Real World Appl. 13 (2012), 419-431.

3. A. Algaba, C. García and M. Reyes, Integrability of two dimensional quasihomogeneous polynomial differential systems, Rocky Mountain J. Math. 41 (2011), $1-22$.

4. $ـ$, Rational integrability of two-dimensional quasi-homogeneous polynomial differential systems, Nonlin. Anal. 73 (2010), 1318-1327. 
5. A. Algaba, C. García and M.A. Teixeira, Reversibility and quasi-homogeneous normal forms of vector fields, Nonlin. Anal. 73 (2010), 510-525.

6. C. Cairó and J. Llibre, Polynomial first integrals for weight-homogeneous planar polynomial differential systems of weight degree 3, J. Math. Anal. Appl. 331 (2007), 1284-1298.

7. J. Chavarriga, H. Giacomini, J. Giné and J. Llibre, Darboux integrability and the inverse integrating factor, J. Diff. Eq. 194 (2003), 116-139.

8. A. Cima and J. Llibre, Algebraic and topological classification of the homogeneous cubic vector fields in the plane, J. Math. Anal. Appl. 147 (1990), 420-448.

9. T. Date and M. Lai, Canonical forms of real homogeneous quadratic transformations, J. Math. Anal. Appl. 56 (1976), 650-682.

10. S.D. Furta, On non-integrability of general systems of differential equations, Z. Angew. Math. Phys. 47 (1996), 112-131.

11. A. Goriely, Integrability, partial integrability, and nonintegrability for systems of ordinary differential equations, J. Math. Phys. 37 (1996), 1871-1893.

12. S. Labrunie, On the polynomial first integrals of the $(a, b, c)$ Lotka-Volterra system, J. Math. Phys. 37 (1996), 5539-5550.

13. J. Llibre and X. Zhang, Polynomial first integrals for quasi-homogeneous polynomial differential systems, Nonlinearity 15 (2002), 1269-1280.

14. A.J. Maciejewski and J.M. Strelcyn, On algebraic non-integrability of the Halphen system, Phys. Lett. 201 (1995), 161-166.

15. J. Moulin-Ollagnier, Polynomial first integrals of the Lotka-Volterra system, Bull. Sci. Math. 121 (1997), 463-476.

16. A. Tsygvintsev, On the existence of polynomial first integrals of quadratic homogeneous systems of ordinary differential equations, J. Phys. Math. Gen. 34 (2001), 2185-2193.

Departament de Matemàtiques, Universitat Autònoma de Barcelona, 08193 Bellaterra, Barcelona, Catalonia, Spain

Email address: jllibre@mat.uab.cat

Departamento de Matemática, Instituto Superior Técnico, 1049-001 Lisboa, Portugal

Email address: cvalls@math.ist.utl.pt 